\title{
CRITICAL SOUND PROPAGATION IN MAGNETIC METALS AND BOTTLENECK PHENOMENA
}

\author{
A. PAWLAK \\ Institute of Physics, A. Mickiewicz University \\ Umultowska 85, 61-614 Poznań, Poland
}

\begin{abstract}
A semiphenomenological model of sound propagation in a critical magnetic system is proposed, which takes into account a relaxation of energy between the system of localised spins and the conduction electron spins as well as the coupling of the longitudinal phonon to bilinear combination of spin fluctuations and to the energy densities. A general expression for the acoustic self-energy is obtained within the one-loop approximation. The effect of "bottlenecking" and its influence on the critical singularities in the sound characteristics is discussed.
\end{abstract}

PACS numbers: 05.70.Jk, 62.65.+k

There are many different regimes in the critical sound attenuation in magnets, depending on sound frequency, reduced temperature and some relevant relaxation frequencies $[1,2]$. In this paper we study an Ising-like, local spin system coupled to the longitudinal sound mode and three kinds of energy densities. In analysis of metal systems, we should take into consideration the relaxation between the three components of the system - the localised spins, conduction electrons, and the lattice. As we shall see, in some circumstances a ratio of the relaxation rates may be such as to modify the phonon self-energy and a kind of bottleneck effect can appear.

We consider a one-component spin (order parameter) $S(x)$ coupled to the local strain $e_{\alpha \beta}(x)$ and three energy densities: the spin energy $e_{\mathrm{S}}(x)$, lattice energy $e_{\mathrm{L}}(x)$ and the spin energy of the conduction electrons $e_{\mathrm{c}}(x)$. The interactions are described by the functional

$$
\begin{aligned}
& H=\frac{1}{2} \int \mathrm{d}^{d} x\left[r S^{2}+(\nabla S)^{2}+\frac{u}{2} S^{4}+C_{12}\left(\sum_{\alpha} e_{\alpha \alpha}\right)^{2}+2 C_{44} \sum_{\alpha, \beta} e_{\alpha \beta}^{2}+\frac{e_{\mathrm{S}}^{2}}{C_{\mathrm{S}}}\right. \\
& \left.+\frac{e_{\mathrm{L}}^{2}}{C_{\mathrm{L}}}+\frac{e_{\mathrm{c}}^{2}}{C_{\mathrm{c}}}+2 g \sum_{\alpha} e_{\alpha \alpha} S^{2}+2 f e_{\mathrm{S}} S^{2}+2 w\left(e_{\mathrm{S}}+a_{1} e_{\mathrm{L}}+a_{2} e_{\mathrm{c}}\right) \sum_{\alpha} e_{\alpha \alpha}\right]
\end{aligned}
$$

where the first three terms in this Hamiltonian compose the Ginzburg-Landau functional for the order parameter. The energy field $e_{\mathrm{L}}(x)$ is related to all vibrations of lattice atoms excluding sound. Elastic degrees of freedom are already accounted (fourth and fifth term of (1)) and for isotropic media $C_{44}=$ $2\left(C_{11}-C_{12}\right)$, where $C_{\alpha \beta}$ are the bare elastic constants. The next three terms form the lowest-order expansion of the functional with respect to energy fields 
with $C_{\mathrm{L}}, C_{\mathrm{S}}$, and $C_{\mathrm{e}}$ as the corresponding bare specific heats. The other terms describe interactions. We postulate that besides the magnetostrictive coupling (with the coupling constant $g$ ) there are also strain-energy density interactions with the coupling constants $w, a_{1} w$, and $a_{2} w$. The sound mode appears in our theory after normal mode expansion of the strain tensor.

The dynamics of the model is described by the nonlinear Langevin equations

$$
\begin{aligned}
& \dot{S}_{\boldsymbol{k}}=-\Gamma \frac{\delta H}{\delta S_{-\boldsymbol{k}}}+\xi_{\boldsymbol{k}}, \\
& \ddot{Q}_{\boldsymbol{k}}=-\frac{\delta H}{\delta Q_{-\boldsymbol{k}}}-\theta k^{2} \dot{Q}_{k}+\eta_{\boldsymbol{k}}, \\
& \dot{e}_{\boldsymbol{k}}^{\mathrm{S}}=-\left(\gamma+\beta+\lambda_{\mathrm{S}} k^{2}\right) \frac{\delta H}{\delta e_{-\boldsymbol{k}}^{\mathrm{S}}}+\gamma \frac{\delta H}{\delta e_{-\boldsymbol{k}}^{\mathrm{L}}}+\beta \frac{\delta H}{\delta e_{-\boldsymbol{k}}^{\mathrm{c}}}+\varphi_{\boldsymbol{k}}, \\
& \dot{e}_{\boldsymbol{k}}^{\mathrm{L}}=-\left(\gamma+\delta+\lambda_{\mathrm{L}} k^{2}\right) \frac{\delta H}{\delta e_{-\boldsymbol{k}}^{\mathrm{L}}}+\gamma \frac{\delta H}{\delta e_{-\boldsymbol{k}}^{\mathrm{S}}}+\delta \frac{\delta H}{\delta e_{-\boldsymbol{k}}^{\mathrm{c}}}+\psi_{\boldsymbol{k}}, \\
& \dot{e}_{\boldsymbol{k}}^{\mathrm{c}}=-\left(\beta+\delta+\lambda_{\mathrm{c}} k^{2}\right) \frac{\delta H}{\delta e_{-\boldsymbol{k}}^{\mathrm{c}}}+\beta \frac{\delta H}{\delta e_{-\boldsymbol{k}}^{\mathrm{S}}}+\delta \frac{\delta H}{\delta e_{-\boldsymbol{k}}^{\mathrm{L}}}+\chi_{k},
\end{aligned}
$$

where the Fourier representation has been used, $Q_{\boldsymbol{k}}$ is the longitudinal phonon coordinate and damping coefficients $\Gamma, \theta k^{2},\left(\gamma+\beta+\lambda_{S} k^{2}\right),\left(\gamma+\delta+\lambda_{\mathrm{L}} k^{2}\right)$, and $\left(\beta+\delta+\lambda_{\mathrm{L}} k^{2}\right)$ are related to the variances of the noises $\xi_{k}, \eta_{k}, \varphi_{\boldsymbol{k}}, \psi_{\boldsymbol{k}}$ and $\chi_{\boldsymbol{k}}$ through the Einstein relations. The last three equations describe the energy flows between the localised spins, lattice and conduction electron subsystems which tend to equalize the local temperatures in the three subsystems. In metals usually $\gamma \ll \beta, \delta$ and direct relaxation from the local spins to the lattice can be neglected.

It is convenient to represent the equations of motion in the functional representation [3] with the Lagrangian given by

$$
\begin{gathered}
L=\int_{\omega} \sum_{k}\left\{\Gamma \tilde{S}_{\boldsymbol{k}, \omega} \tilde{S}_{-\boldsymbol{k},-\omega}+\theta k^{2} \tilde{Q}_{\boldsymbol{k}, \omega} \tilde{Q}_{-\boldsymbol{k},-\omega}+\Gamma_{\mathrm{S}}(k) \tilde{e}_{\boldsymbol{k}, \omega}^{\mathrm{S}} \tilde{e}_{-\boldsymbol{k},-\omega}^{\mathrm{S}}+\Gamma_{\mathrm{L}}(k)\right. \\
\times \tilde{e}_{\boldsymbol{k}, \omega}^{\mathrm{L}} \tilde{e}_{-\boldsymbol{k},-\omega}^{\mathrm{L}}+\Gamma_{\mathrm{c}}(k) \tilde{e}_{\boldsymbol{k}, \omega}^{\mathrm{c}} \tilde{e}_{-\boldsymbol{k},-\omega}^{\mathrm{c}}-2 \gamma \tilde{e}_{\boldsymbol{k}, \omega}^{\mathrm{S}} \tilde{e}_{-\boldsymbol{k},-\omega}^{\mathrm{L}}-2 \beta \tilde{e}_{\boldsymbol{k}, \omega}^{\mathrm{S}} \tilde{e}_{-\boldsymbol{k},-\omega}^{\mathrm{c}}-2 \delta \tilde{e}_{\boldsymbol{k}, \omega}^{\mathrm{c}} \tilde{e}_{-\boldsymbol{k},-\omega}^{\mathrm{L}} \\
-\tilde{Q}_{\boldsymbol{k}, \omega}\left[\left(-\omega^{2}+\mathrm{i} \theta k^{2} \omega\right) Q_{-\boldsymbol{k},-\omega}+\frac{\partial H}{\partial Q_{\boldsymbol{k}, \omega}}\right]-\tilde{S}_{\boldsymbol{k}, \omega}\left(\mathrm{i} \omega S_{-\boldsymbol{k},-\omega}+\Gamma \frac{\partial H}{\partial S_{\boldsymbol{k}, \omega}}\right) \\
-\tilde{e}_{\boldsymbol{k}, \omega}^{S}\left[\mathrm{i} \omega e_{-\boldsymbol{k},-\omega}^{\mathrm{S}}+\Gamma_{\mathrm{S}}(k) \frac{\partial H}{\partial e_{\boldsymbol{k}, \omega}^{\mathrm{S}}}-\gamma \frac{\partial H}{\partial e_{\boldsymbol{k}, \omega}^{\mathrm{L}}}-\beta \frac{\partial H}{\partial e_{\boldsymbol{k}, \omega}^{\mathrm{c}}}\right] \\
-\tilde{e}_{\boldsymbol{k}, \omega}^{\mathrm{L}}\left[i \omega e_{-\boldsymbol{k},-\omega}^{\mathrm{L}}+\Gamma_{\mathrm{L}}(k) \frac{\partial H}{\partial e_{\mathbf{k}, \omega}^{\mathrm{L}}}-\gamma \frac{\partial H}{\partial e_{\boldsymbol{k}, \omega}^{\mathrm{S}}}-\delta \frac{\partial H}{\partial e_{\boldsymbol{k}, \omega}^{\mathrm{c}}}\right] \\
\left.-\tilde{e}_{\boldsymbol{k}, \omega}^{\mathrm{c}}\left[i \omega e_{-\boldsymbol{k},-\omega}^{\mathrm{c}}+\Gamma_{\mathrm{c}}(k) \frac{\partial H}{\partial e_{\boldsymbol{k}, \omega}^{\mathrm{c}}}-\beta \frac{\partial H}{\partial e_{\boldsymbol{k}, \omega}^{\mathrm{S}}}-\delta \frac{\partial H}{\partial e_{\boldsymbol{k}, \omega}^{\mathrm{L}}}\right]\right\}
\end{gathered}
$$


where $\tilde{S}, \tilde{Q}, \tilde{e}_{\mathrm{S}}, \tilde{e}_{\mathrm{L}}$, and $\tilde{e}_{\mathrm{c}}$ are auxiliary "response" fields, $\Gamma_{i}(k)=\gamma_{i}+\lambda_{i} k^{2}$ for $i=\mathrm{S}, \mathrm{L}, \mathrm{c}$ and $\gamma_{\mathrm{S}}=\gamma+\beta, \gamma_{\mathrm{L}}=\gamma+\delta$ and $\gamma_{\mathrm{c}}=\beta+\delta$.

With the aid of the Gaussian transformation

$$
\begin{aligned}
& e_{\mathrm{L}} \rightarrow e_{\mathrm{L}}+\mathcal{A} e_{\mathrm{S}}+\mathcal{B} e_{\mathrm{c}}, \\
& \tilde{e}_{\mathrm{L}} \rightarrow \tilde{e}_{\mathrm{L}}+\mathcal{C} \tilde{e}_{\mathrm{S}}+\mathcal{D} \tilde{e}_{\mathrm{c}}+\mathcal{E} e_{\mathrm{S}}+\mathcal{F} e_{\mathrm{c}},
\end{aligned}
$$

where $\mathcal{A}, \mathcal{B}, \ldots$ are frequency dependent coefficients, the lattice energy field can be decoupled from the other energies. The free spin-energy and conduction electron-energy response function get renormalised to the form

$$
\begin{aligned}
& \tilde{D}_{\mathrm{S} 0}^{-1}(k, \omega)=D_{\mathrm{S} 0}^{-1}(k, \omega)-\frac{\gamma^{2}}{\Gamma_{\mathrm{S}}(k) C_{\mathrm{S}} \Gamma_{\mathrm{L}}(k)\left(1-\mathrm{i} \bar{\omega}_{\mathrm{L}}\right)} \\
& \tilde{D}_{\mathrm{c} 0}^{-1}(k, \omega)=D_{\mathrm{c} 0}^{-1}(k, \omega)-\frac{\delta^{2}}{\Gamma_{\mathrm{c}}(k) C_{\mathrm{c}} \Gamma_{\mathrm{L}}(k)\left(1-\mathrm{i} \bar{\omega}_{\mathrm{L}}\right)},
\end{aligned}
$$

where $D_{\mathrm{S} 0}(k, \omega)=\left(-\mathrm{i} \omega / \Gamma_{\mathrm{S}}+C^{-1} \mathrm{~s}\right)^{-1}, D_{\mathrm{c} 0}(k, \omega)=\left(-\mathrm{i} \omega / \Gamma_{\mathrm{c}}+C_{\mathrm{c}}^{-1}\right)^{-1}$ and $\bar{\omega}_{\mathrm{L}}=\omega / \omega_{\mathrm{L}}$ is the frequency reduced with respect to the bare lattice-energy relaxation rate $\omega_{\mathrm{L}}=\Gamma_{\mathrm{L}} / C_{\mathrm{L}}$. Assuming the adiabatic approximation for the lattice, $\bar{\omega}_{\mathrm{L}} \gg 1$, which is justified for $C_{\mathrm{L}} \gg C_{\mathrm{S}}, C_{\mathrm{c}}$ we obtain $\tilde{D}_{\mathrm{SO}} \approx D_{\mathrm{S} 0}$ and $\tilde{D}_{\mathrm{c} 0} \approx D_{\mathrm{c} 0}$.

Next, using similar Gaussian transformations we decouple the sound mode from the spin and energy fields getting a general expression for the acoustic self-energy [2]

$$
\frac{\Sigma(k, \omega)}{k^{2}}=\frac{2\left[g^{2}(k, \omega)-\mathrm{i} \tilde{\omega} b(k, \omega) g^{2}\right] \Psi+w^{2} P^{2} C_{\mathrm{S}}}{1-\mathrm{i} \tilde{\omega} b(k, \omega)(1+v \Psi)},
$$

where $\Psi=\left\langle\widetilde{\Gamma S^{2}}-\boldsymbol{k},-\omega S_{\vec{k}, \omega}^{2}\right\rangle^{L_{A}}, g(k, \omega)=g-w f C_{\mathrm{S}} P(k, \omega)$ is an effective frequency-dependent coupling constant with $P(k, \omega)=\frac{M-i \bar{\omega}\left(1-a_{2} \beta / \Gamma_{\mathrm{S}}\right)}{M-\mathrm{i} \bar{\omega}}, \tilde{\omega}=\omega C_{\mathrm{S}} / \Gamma_{\mathrm{S}}(k)$, and $\bar{\omega}=\omega C_{\mathrm{c}} / \Gamma_{\mathrm{c}}(k)$. The four-spin response function $\Psi$ is calculated with the effective, phonon and energy free Lagrangian of the model $A$ in the classification of Hohenberg and Halperin $[4] ; b(q, \omega)=\frac{1-\mathrm{i} \bar{\omega}}{M-\mathrm{i} \bar{\omega}}$ is a coefficient containing details of dynamics. The parameter $M=1-\frac{\beta^{2}}{\Gamma_{\mathrm{S}}(k) \Gamma_{\mathrm{c}}(k)} \approx \frac{\delta}{\delta+\beta}$ takes a value close to unity in the normal (unbottlenecked) case when $\delta \gg \beta$, i.e. when the conduction electrons transfer their energy much faster to the lattice than to the local-spin system. When $\delta \ll \beta$ i.e. the conduction electron-lattice relaxation rate is slow and energy is "piling up" in the conduction electrons spin system, one can study, by analogy to the electron spin resonance phenomena in metals [5], a bottleneck effect in the sound propagation. In that case $M \approx \delta / \beta \ll 1$ and for low frequencies we get

$$
\frac{\Sigma(k, \omega)}{k^{2}} \approx \frac{2\left[\left(g-w f C_{\mathrm{S}}\right)^{2}-\frac{\mathrm{i} \tilde{\omega}}{M} g^{2}\right] \Psi}{1-\frac{\mathrm{i} \tilde{\omega}}{M}(1+v \Psi)}
$$

which is analogous to the expression for the normal system, where instead of $\tilde{\omega} / M$ only $\tilde{\omega}$ appears. As $M \ll 1$ for the bottlenecked systems, the role of the denominator in Eq. (11) is much more important than for normal systems. As a consequence, 
the sound attenuation coefficient, which is determined by the imaginary part of $\Sigma$, is given by

$$
\alpha(\omega, t) \approx\left(g-w f C_{\mathrm{S}}\right)^{2} \omega^{2}\left[W_{1} t^{-(z \nu+\alpha)} \frac{\operatorname{Im} \Phi(y)}{y}+W_{2} t^{-2 \alpha}|\Phi(y)|^{2}\right],
$$

where the scaling relation $\Psi=t^{-\alpha} \Phi(y)$ has been used with $\Phi(y)$ as a scaling function, $y=\omega t^{-z \nu} / \Gamma$ as the reduced frequency and $t=\left(T-T_{\mathrm{C}}\right) / T_{\mathrm{C}}$ as the reduced temperature. In Eq. (12) strong singularity (the first term), called also the Murata-Iro-Schwabl singularity, competes with the weak singularity (the Kawasaki term), where the singularity exponent $\rho=2 \alpha$ is very small, like in magnetic insulators [6]. What is important, the relative weight of both terms $W_{2} / W_{1}$ is increased in the bottlenecked system by the factor of $M^{-1} \approx \beta / \delta \gg 1$ in comparison with that in the normal system. In principle, changing the amount of magnetic impurities or adding some other impurities, we are able to change the conduction electron-lattice relaxation rate. By lowering $\delta$, a crossover to the weak singularity (insulator-like behaviour) should be observed which is consequence of the bottlenecking of the system.

For high frequencies the acoustic self-energy takes the form

$$
\frac{\Sigma(k, \omega)}{k^{2}} \approx 2 \frac{\left[\left(g-w f C_{\mathrm{S}}\left(1-a_{2}\right)\right)^{2} C_{\mathrm{c}}+g^{2} C_{\mathrm{S}}-\mathrm{i} \tilde{\omega} g^{2} C_{\mathrm{c}}\right] \Psi}{C_{\mathrm{c}}+C_{\mathrm{S}}(1+v \Psi)-\mathrm{i} \tilde{\omega} C_{\mathrm{c}}(1+v \Psi)} .
$$

The only difference between normal and bottlenecked system is that the high-frequencies for the former means $\bar{\omega} \gg 1$ and for the latter $\bar{\omega} \gg M$, so the high-frequency regime begins, for the bottlenecked system, from much lower frequencies than for the normal system. The sound attenuation coefficient is dominated here by the singularity of different type

$$
\alpha(\omega, t) \propto g^{2} \omega^{2} t^{-(z \nu-\alpha)} \frac{\operatorname{Im} \Phi(y)^{-1}}{y},
$$

where $C_{\mathrm{c}} \ll C_{\mathrm{S}}$ was assumed. The high-frequency behaviour of $\alpha(\omega, t)$, can be also called "fully adiabatic" behaviour [2] as the local spin system and the conduction electron spin system no longer can be treated as at equilibrium with sound wave or with each other. Thus, changing $\delta$ we are able also, at least in principle, to shift the adiabatic region towards lower frequencies making it possible to be observed also in the ultrasonic frequency range.

\section{References}

[1] A. Pawlak, Eur. Phys. J. B 4, 179 (1998).

[2] A. Pawlak, preprint a vailable at: http:/xxx.lanl.gov/cond-mat/9903229.

[3] R. Bausch, H.K. Janssen, H. Wagner, Z. Phys. B 24, 113 (1976).

[4] P.C. Hohenberg, B.I. Halperin, Rev. Mod. Phys. 49, 435 (1977).

[5] R.H. Taylor, Adv. Phys. 24, 681 (1975).

[6] B. Lüthi, in: Dynamical Properties of Solids, Eds. G.K. Horton, A.A. Marudin, Vol. 3, North-Holland, Amsterdam 1980, p. 245. 